\title{
Recurrent Skin Angiosarcoma
}

National Cancer Institute

\section{Source}

National Cancer Institute. Recurrent Skin Angiosarcoma. NCI Thesaurus. Code C153626.

The reemergence of skin angiosarcoma after a period of remission. 